\title{
CORRECTION
}

\section{Correction: A survey of community search over big graphs}

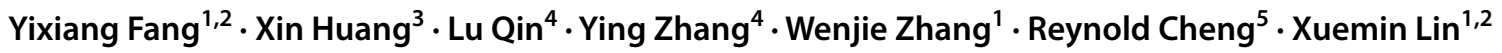

Published online: 11 November 2019

(c) Springer-Verlag GmbH Germany, part of Springer Nature 2019

\section{Correction to: The VLDB Journal https://doi.org/10.1007/s00778-019-00556-x}

In the original article, the Table 1 was published with incorrect figures. The correct Table 1 is given below:

Table 1 Classification of works of community search ("P." means Problem)

\begin{tabular}{|c|c|c|c|c|c|c|}
\hline \multirow[t]{2}{*}{ Metric } & \multirow[t]{2}{*}{ Simple graphs } & \multicolumn{5}{|c|}{ Attributed graphs } \\
\hline & & Keyword & Location & Temporal & Influence (weight) & Profile \\
\hline k-core & $\begin{array}{l}{[15,46,66,175]} \\
(\text { P. } 1,2,3,4,5)\end{array}$ & $\begin{array}{l}{[58,61]} \\
\text { (P. 6) }\end{array}$ & $\begin{array}{l}{[60,65,185,221]} \\
(\text { P. } 7,8,9)\end{array}$ & $\begin{array}{l}{[129]} \\
(\text { P. 10) }\end{array}$ & $\begin{array}{l}{[21,30,126-128,215]} \\
\text { (P. } 11,12,13)\end{array}$ & $\begin{array}{l}{[31]} \\
\text { (P. 14) }\end{array}$ \\
\hline k-truss & $\begin{array}{l}{[6,98,101]} \\
(\mathrm{P} .15,16)\end{array}$ & $\begin{array}{l}{[102]} \\
(\mathrm{P} .17)\end{array}$ & - & - & $\begin{array}{l}{[216]} \\
(\text { P. 18) }\end{array}$ & - \\
\hline k-clique & $\begin{array}{l}{[45,187,195,205]} \\
(\text { P. } 19,20,21,22)\end{array}$ & - & - & $\begin{array}{l}{[125]} \\
(\text { P. 23) }\end{array}$ & - & - \\
\hline k-ECC & $\begin{array}{l}{[25,95,96]} \\
\text { (P. } 24,25,26)\end{array}$ & - & - & - & - & - \\
\hline Others & \multicolumn{6}{|c|}{$\begin{array}{l}\text { Local modularity: [40,136] query biased density: [190] pagerank: [9,114] (P. 27) neighbors: } \\
\text { [142] }\end{array}$} \\
\hline
\end{tabular}

Publisher's Note Springer Nature remains neutral with regard to jurisdictional claims in published maps and institutional affiliations.

The original article can be found online at https://doi.org/10.1007/ s00778-019-00556-x.

Yixiang Fang

yixiang.fang@unsw.edu.au

Xin Huang

xinhuang@comp.hkbu.edu.hk

Lu Qin

lu.qin@uts.edu.au

Ying Zhang

ying.zhang@uts.eud.au

Wenjie Zhang

zhangw@cse.unsw.edu.au

Reynold Cheng

ckcheng@cs.hku.hk
Xuemin Lin

1xue@cse.unsw.edu.au

University of New South Wales, Sydney, Australia

2 Zhejiang Lab, Hangzhou, China

Hong Kong Baptist University, Kowloon Tong, Hong Kong

4 The University of Technology Sydney, Sydney, Australia

5 The University of Hong Kong, Pok Fu Lam, Hong Kong 